\title{
Phytochemical Components of Two Cyanobacterial Local Strains
}

\author{
Mohamed A. Deyab ${ }^{1}$, Mostafa M. El-Sheekh², Reham S. A. Hasan ${ }^{3}$, Abdelgawad Y. Elsadany ${ }^{3}$ and Seham E. \\ Abu Ahmed*1 \\ ${ }^{1}$ Batony Department, Faculty of Science, Demietta University, Egypt. \\ ${ }^{2}$ Botany Department, Faculty of Science, Tanta University, Egypt. \\ ${ }^{3}$ Cyanobacteria Lab., Microbiology Dept., Soils, Water and Environment Research Institute, Sakha Agriculture \\ Research Station, ARC, Giza, Egypt.
}

Received: 10 April 2021 /Accepted: 16 April 2021

*Corresponding author's E-mail: Dr_Seham2016@yahoo.com

\begin{abstract}
Anabaena variabilis was collected by isolation from saline soil at Elhamol city, Kafr Elsheikh governerate, Egypt. Spirulina platensis was obtained from cyanobacteria Lab., Microbiology Dept., Sakha Agricultural Research Station, Egypt. GC/MS analysis showed that 37 and 7 bioactive compounds from A. variabilis and S. platensis were identified, respectively. Moreover, the area percentage of the same bioactive compound varied according to the type of cyanobacteria. The major phytochemical constituents in the methanol extract of A. variabilis are Pentadecanoic acid 29\%, 12,15-Octadecadienoic acid methyl ester $10.26 \%$, Hexadecenoic acid methyl ester (Z) $8.3 \%$, Thieno [3,4-C] pyridine, 1,3,4,7-tetraphenyl $5.86 \%$. While, S. platensis methanol extract contain Hexadecanoic acid 29\%, 9,12,15-Octadecadienoic acid methyl ester 24\% and 9,12 Octadecatrienoic acid zz methyl ester, $24.36 \%$.
\end{abstract}

Keywords: Cyanobacteria, GC/MS analysis, Phytocomponents, saline soil.

\section{Introduction}

Cyanobacteria are photosynthetic Gramnegative prokaryotes and can grow in a variety of aquatic and terrestrial environments (Harjinder et al. 2014). Cyanobacteria are known to be the spearhead organisms in major habitats and along with their diversity in structure and ability to supply a variety of compounds as pigments, vitamins, and enzymes (Seddek et al. 2019). Very few studies have isolated the cyanobacteria from El-Hamoul.
Maybe climate changes affect the structure and composition of cyanobacterial communities which may lead to a change in the phytochemical composition of cyanobacteria. There are possibility of using these strains in the pharmaceutical industry and agriculture. Anabaena variabilis, Nostoc muscorum, Nostoc linckia, Oscillatoria acuminata, Oscillatoria amphigranulata and Spirulina platensis were isolated from different regions of paddy fields in Nile Delta, Egypt and these isolates have different bioactive compounds (Gheda and Ismail 2020). The presence of secondary or primary metabolites of varieties bioactive 
compounds within the plant and cyanobacteria recommended using them for phytopharmaceutical importance (Prakash $\boldsymbol{e t}$ al. 2011; Varsha and Meeta 2019). Cyanobacteria used as antioxidants, anticancer, antiviral properties used in agriculture, industry, medicine, biotechnology, and pharmaceutical fields (Patra $\boldsymbol{e t}$ al. 2008), and antimicrobial that can inhibit or kill pathogenic microorganisms. The mode of action of these antimicrobial compounds is modifications of the structure, function, or damage of the cytoplasmic membrane, destruction of enzymes, and suppression of protein synthesis (Swain et al. 2017). The activity of antimicrobial depends on the specie of alga and the type of used solvents (Radhika et al. 2012). Cyanobacteria produce natural products, which increase the ability to survive in a variety of environmental stress. Natural products are used in disease control for decades. Cyanobacteria have new drugs to treat incurable diseases (Bethan and Carole 2018).

Modern advances in biotechnology are geared towards increasing the production of desired products in cyanobacteria to be used for various industrial applications (Abed et al. 2011; Rama-Murthy et al. 2012; Lau et al. 2015). Cyanobacteria produce several biocidal metabolites, including antitumor activity (Yadav et al. 2016; Camila et al. 2018), toxins (Agrawal, et al. 2006), and enzyme inhibitors (Skulberg, 2000). These metabolites have many functions that can be helpful in pathogen control against predators, chemosensory, and photo-protection. These properties can be utilized in industrial biotechnology as nutraceuticals, cosmeceuticals, and pharmaceuticals, due to adaptation mechanisms of microorganisms against the action of antimicrobial drugs (Elshouny et al. 2017), which considered the main reason for continued research into antimicrobial compounds (AlWathnani et al. 2012). The present work aims to study the phytochemical components of two cyanobacterial strains isolated from soil and aquatic habitat, identify the chemical constituents for possible exploitation in industry and other application.

\section{Materials and methods}

\section{Cyanobacterial cultivation}

Anabaena variabilis was collected by isolation from saline soil at Elhamol city, Kafr Elsheikh governerate, Egypt. Spirulina platensis was obtained from cyanobacteria Lab., Microbiology Dept., Sakha Agricultural Research Station, Egypt. Cyanobacterial species was purified and grown in $100 \mathrm{ml}$ sterilized BG-11 nitrogen-free medium, $\mathrm{pH}$ 7.5, and incubated under illumination (5000 Lux) at $28 \pm 2{ }^{\circ} \mathrm{C}$. After 20 days, the cyanobacterial strains were transferred to $6 \mathrm{~L}$ transparent glass bottles , containing 4L BG-11 medium inoculated with $100 \mathrm{ml}$ of pre-cultured strains. The flasks were aerated with an air pump at regular pressure.

\section{Preparation of cyanobacterial methanol extracts}

The cyanobacterial biomass from the exponential growth phase was centrifuged at $10,000 \mathrm{rpm}$ for $3 \mathrm{~min}$. then filtered through filter paper (Wattman-4) and air-dried (Starr $\boldsymbol{e t}$ al. 1962). For extraction, the dried cell mass of cyanobacteria was extracted by dissolving in methanol $(1 \mathrm{~g} / 10 \mathrm{ml})$ and kept overnight for complete extraction. The supernatant was separated by filter with $0.5 \mu \mathrm{m}$ pore size, the dry residue was re-dissolved in dimethyl-sulfoxide (DMSO), and this is called the crude extract $(100 \%)$. Then they were kept in fresh glass vials in the dark at $4^{\circ} \mathrm{C}$ till using for phytochemical screening by GC mass (Lefort et al. 1988).

\section{Gas chromatography -Mass spectrophotometry}

Profile of metabolite has been developed as a new technology platform in biological samples that describes complex chemical matrices and identifies the compounds. In particular, GC/MS is a fast and precise tool commonly used in diagnostics, functional genomics, and screening (Rohloff 2015). The GC/MS is a direct and fast analytical method for the identification of cyanobacterial extracts components. Extracts of Anabaena variabilis and Spirulina platensis were performed using Trace GC-TSQ Quantum mass spectrometer (Thermo Scientific, Austin, TX, USA) with a direct capillary column TG-5MS (30 m x 0.25 $\mathrm{mm} \times 0.25 \mu \mathrm{m}$ film thickness). The temp of the column oven was initially held at $50^{\circ} \mathrm{C}$ and then increased by $5^{\circ} \mathrm{C} / \mathrm{min}$ to $200^{\circ} \mathrm{C}$ hold for $2 \mathrm{~min}$. Increased to the final temperature of $290^{\circ} \mathrm{C}$ by 
$30^{\circ} \mathrm{C} / \mathrm{min}$ and hold for $2 \mathrm{~min}$. The injector and MS transfer line temperatures were kept at 270, $260^{\circ} \mathrm{C}$, respectively. The carrier gas was helium at a constant flow rate of $1 \mathrm{ml} / \mathrm{min}$.

\section{Results and discussion}

Physical and chemical characteristics, of the collected soil samples were presented in Table 1. There is a large variation between those samples in electric conductivity (E.C), which ranged from 7.75 to $15.93 \mathrm{dS} . \mathrm{m}^{-1}$. Soils were classified as saline soil. Saline soils have traditionally been classified as those in which the EC is higher than $4 \mathrm{dS} \mathrm{m}^{-1}$. The major problem with saline soils is the presence of soluble salts, primarily $\mathrm{Cl}^{-}, \mathrm{SO}_{4}{ }^{2-}$ (Richards, 1954). In saline soils, the concentration of soluble chloride ions is highest among anion concentrations. Following the chloride ion are sulfate ions, which are followed by bicarbonate ions (Cao et al. 2012). Sodium ion concentration is also increased with an increase in soil salinity (Weggler-Beaton et al. 2000).

Table 1: Physical and chemical characteristics of soil samples.

\begin{tabular}{lcc}
\hline Variables & \multicolumn{2}{c}{ El-Hamoul city } \\
\cline { 2 - 3 } & Site 1 & Site 2 \\
\hline Sand \% & 7.13 & 8.65 \\
Silt \% & 32.43 & 34.95 \\
Clay\% & 60.34 & 56.40 \\
Texture & Clay & Clay \\
pH & 7.93 & 7.87 \\
EC, dS.m & 15.93 & 7.75 \\
Mg+2 & 34.43 & 23.92 \\
$\mathrm{Ca}+2$ & 33.50 & 21.44 \\
$\mathrm{Na}+$ & 90.50 & 30.60 \\
$\mathrm{~K}+$ & 1.50 & 1.54 \\
$\mathrm{SO} 4-2$ & 69.14 & 43.58 \\
$\mathrm{HCO} 3$ & 4.69 & 3.12 \\
$\mathrm{Cl}-$ & 86.10 & 31.80 \\
\hline
\end{tabular}

\section{Cyanobacterial species}

The cyanobacterial species Anabaena variabilis and Spirulina platensis were described in Fig 2 and 3, respectively .These pictures were taken by the OPTICA microscope (Italy) fitted with a Canon Powershot G12 digital camera (El-Gamal et al. 2008).

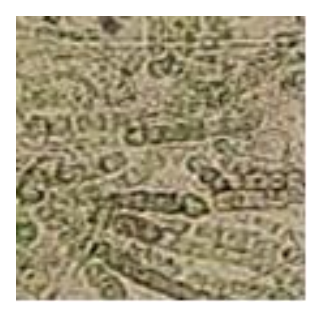

Fig. 2 Anabaena variabilis

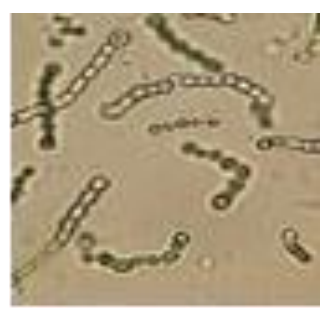

Fig. 3 Spirulina platensis

\section{GC/MS analysis of methanol extract}

The present comparative study contributes valuable information on the bioactive compounds in A. variabilis and $S$. platensis, as summarized in Tables 2 and 3. Numerous bioactive compounds which belong to different classes as fatty acids, phenolic, antioxidants, alkaloids, flavonoids, and steroids were recorded.

Table 2: Methanolic extract components of Anabaena variabilis

\begin{tabular}{llllll}
\hline $\mathbf{N}$ Compound Name \\
\hline 1 Thieno [3,4-c]pyridine, 1,3,4,7- \\
tetraphenyl \\
$\begin{array}{l}\text { Cholan-24-oic acid, 3,7,12-trihydroxy- } \\
\text { (3à,5á,7à,12à)- }\end{array}$
\end{tabular}


3 19-Norethindrone, O-methyloxime

4 Glafenin

5 Androstane-11,17-dione,3[(trimethylsilyl)oxy]-,17-[o(phenylmethyl)oxime], (3à,5à)-

6 Hexasiloxane, $1,3,3,5,5,7,7,9,9,11,11-$ dodecamethyl-

$7 \quad$ Cyclooctasiloxane, hexadecamethyl( Hexadecamethyl cyclooctasioxane)

8 Trimethylsilyl, 3-methyl4[(trimethylsilyl)oxy] Benzoate

9 1H-purin -6-amine, (2fluorophenyl)methyl]

10 Nonadecane(AI3-36122)

11 Heptadecane(AI3-36898)

12 2,7-diphenyl-1,6dioxopyridazino[4,52',3']pyrrolo[4',5'd]pyridazine

13 8,11-Eicosadienoic acid, methyl ester

14 4H-1-benzopyran -4-one, 2-(3,4-dimethoxyphenyl)-3,5-dihydroxy-7methoxy -(3',4',7-trimethylquercetin)

15 18,19-Secoyohimban-19-oic acid, 6,17,20,21-tetradehydro-16(hydroxymethyl)-, methyl ester, (15á,16E)-

16 Benzene, 1,1'-(3,3-dimethyl-1butenylidene)bis-(1-Butene, 3,3dimethyl-1,1-diphenyl)

17 Allopregnane-3á,7à,11à-triol-20-one (3 ,7,11-Trihydroxypregnan-20-one)

18 Hexadecanoic acid, methyl ester (Palmitic acid, methyl ester)
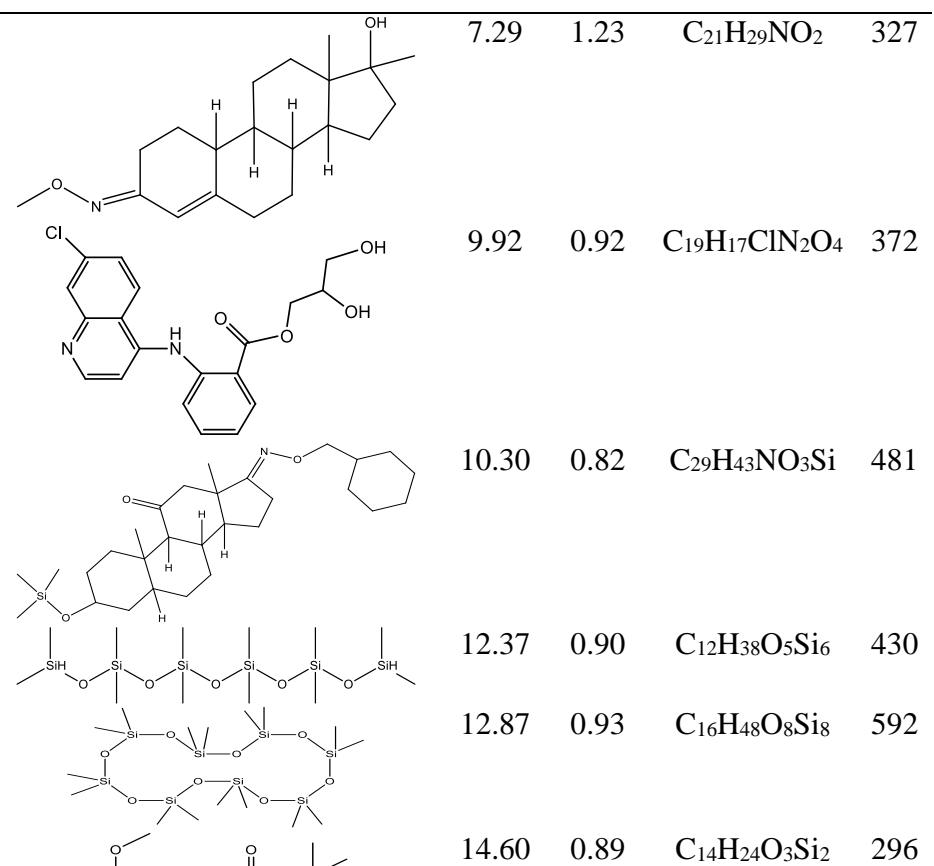

$\begin{array}{llll}7.29 & 1.23 & \mathrm{C}_{21} \mathrm{H}_{29} \mathrm{NO}_{2} & 327\end{array}$

$9.92 \quad 0.92 \quad \mathrm{C}_{19} \mathrm{H}_{17} \mathrm{ClN}_{2} \mathrm{O}_{4} \quad 372$

$\begin{array}{llll}10.30 & 0.82 & \mathrm{C}_{29} \mathrm{H}_{43} \mathrm{NO}_{3} \mathrm{Si} & 481\end{array}$

$\begin{array}{llll}12.37 & 0.90 & \mathrm{C}_{12} \mathrm{H}_{38} \mathrm{O}_{5} \mathrm{Si}_{6} \quad 430\end{array}$

$\begin{array}{llll}12.87 & 0.93 & \mathrm{C}_{16} \mathrm{H}_{48} \mathrm{O}_{8} \mathrm{Si}_{8} \quad 592\end{array}$

$\begin{array}{llll}14.60 & 0.89 & \mathrm{C}_{14} \mathrm{H}_{24} \mathrm{O}_{3} \mathrm{Si}_{2} \quad 296\end{array}$
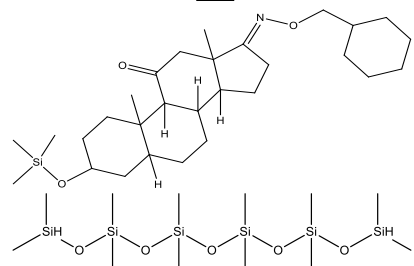<smiles>CC1(C)CC(C)(C)OC(C)(C)OC(C)(C)OC(C)(C)OC(C)(C)O1</smiles><smiles>COC1CCCC(C(=O)OCC(=O)ON(C)C)C1OC(C)(C)C</smiles>

$15.14 \quad 0.55 \quad \mathrm{C}_{12} \mathrm{H}_{10} \mathrm{FN}_{5} \quad 243$<smiles>NC1NC=NC2=NC(Cc3ccccc3F)=NC21</smiles>

$\begin{array}{llll}16.68 & 5.69 & \mathrm{C}_{19} \mathrm{H}_{40} & 268\end{array}$

$\begin{array}{llll}17.23 & 4.85 & \mathrm{C}_{17} \mathrm{H}_{36} & 240\end{array}$

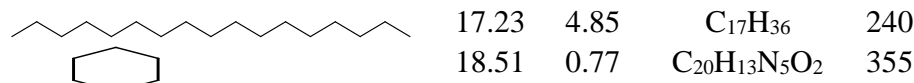

$19.21 \quad 1.27 \quad \mathrm{C}_{21} \mathrm{H}_{38} \mathrm{O}_{2} \quad 322$

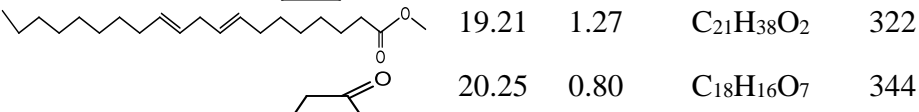

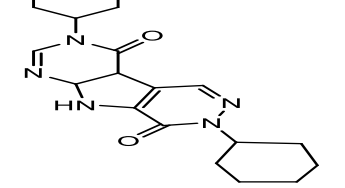

$\begin{array}{llll}20.25 & 0.80 & \mathrm{C}_{18} \mathrm{H}_{16} \mathrm{O}_{7} & 344\end{array}$

$20.34 \quad 0.71 \quad \mathrm{C}_{21} \mathrm{H}_{24} \mathrm{~N}_{2} \mathrm{O}_{3} \quad 352$

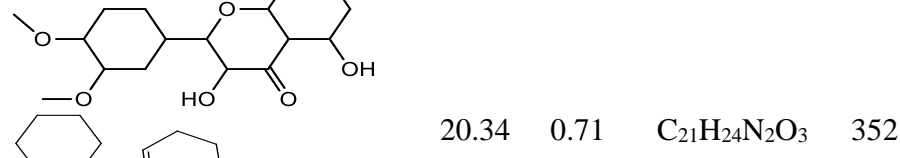

$20.47 \quad 0.48 \quad \mathrm{C}_{18} \mathrm{H}_{20} \quad 236$<smiles>CC(C)(C)C=C(C1=CCCCCC1)c1ccccc1</smiles>

$\begin{array}{llll}21.03 & 0.77 & \mathrm{C}_{21} \mathrm{H}_{34} \mathrm{O}_{4} & 350\end{array}$

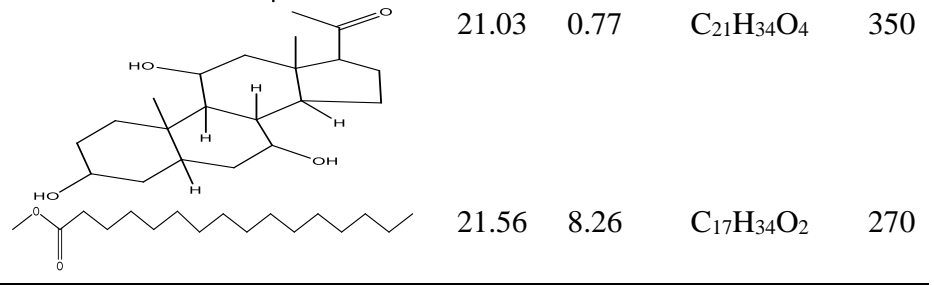




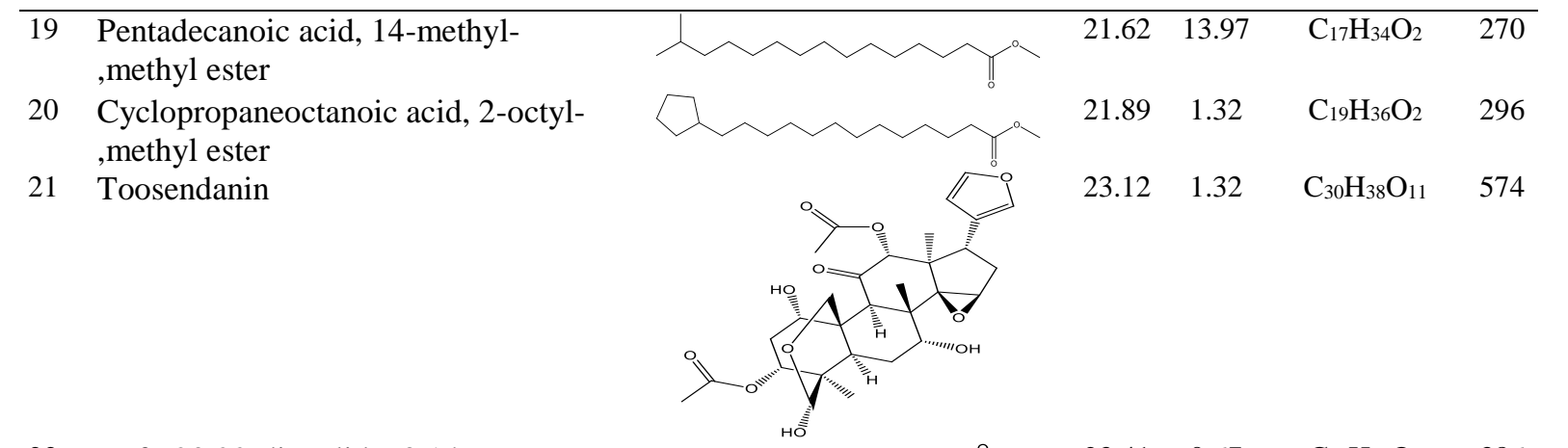

22 Bufa-20,22-dienolide, 3,14dihydroxy-, (3á,5á)5á-Bufa-20,22-dienolide, 3á,14dihydroxy

23 12,15-Octadecadienoic acid, methyl ester

24 Linoleic acid ethyl ester (Ethyl linoleate)

25 9,12-octadeccadienoic acid (Z,Z)-, 2,3dihydroxypropyl ester ( Linolein, 2-mono-)

26 7,10,13-Eicosatrienoic acid, methyl ester

27 12-desoxyphorbol-13-isobutyrat

28 Cyclopropaneoctanoic acid,2[(2pentylcyclopropyl)methyl]-, methyl ester

29 7,8,3',4'-Tetramethoxyflavone

30 Sarreroside

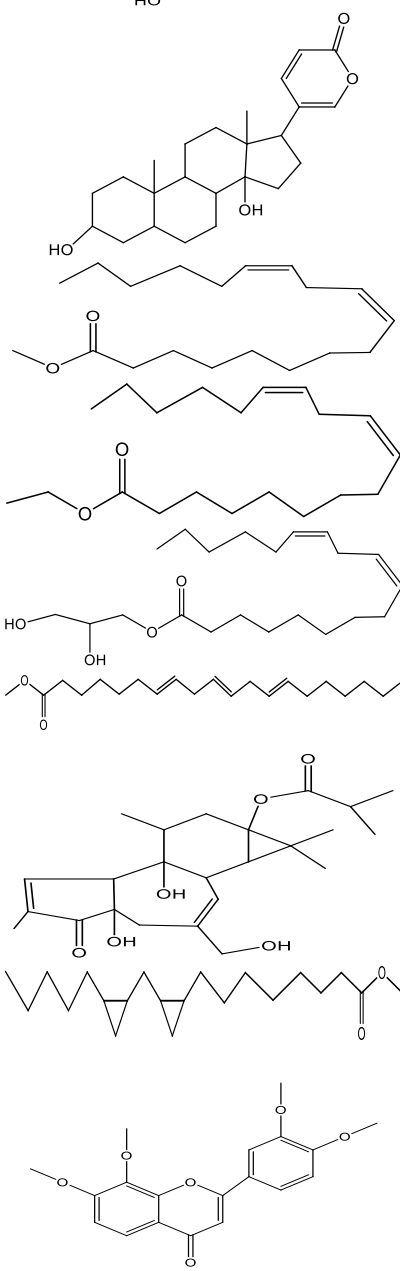

$23.41 \quad 0.67 \quad \mathrm{C}_{24} \mathrm{H}_{34} \mathrm{O}_{4} \quad 386$

$24.41 \quad 10.23 \quad \mathrm{C}_{19} \mathrm{H}_{34} \mathrm{O}_{2} \quad 294$

$24.86 \quad 0.40 \quad \mathrm{C}_{20} \mathrm{H}_{36} \mathrm{O}_{2} \quad 308$

$\begin{array}{llll}25.56 & 2.04 & \mathrm{C}_{21} \mathrm{H}_{38} \mathrm{O}_{4} & 354\end{array}$

$25.97 \quad 1.80 \quad \mathrm{C}_{21} \mathrm{H}_{36} \mathrm{O}_{2} \quad 320$

$26.09 \quad 1.28 \quad \mathrm{C}_{24} \mathrm{H}_{34} \mathrm{O}_{6} \quad 418$

$\begin{array}{llll}26.22 & 0.59 & \mathrm{C}_{21} \mathrm{H}_{38} \mathrm{O}_{2} & 322\end{array}$

$27.54 \quad 0.45 \quad \mathrm{C}_{19} \mathrm{H}_{18} \mathrm{O}_{6} \quad 342$

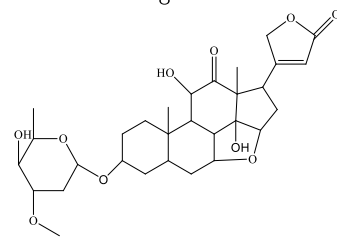

$28.95 \quad 1.32 \quad \mathrm{C}_{30} \mathrm{H}_{42} \mathrm{O}_{10} \quad 562$

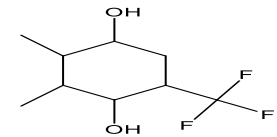

$30.74 \quad 1.23 \quad \mathrm{C}_{9} \mathrm{H}_{9} \mathrm{~F}_{3} \mathrm{O}_{2} \quad 206$

trifluoromethyl-

32 Oxiraneoctanoic acid,3-octyl-, methylester,trans (methyl 8-(3-octyl-2 oxranyl)octanoate)

33 9,10-Secocholesta-5,7,10(19)-triene,25,26-triol

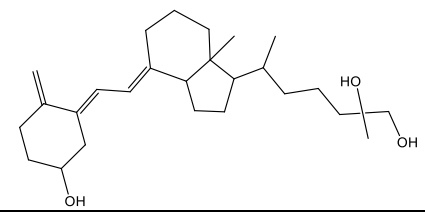

$\begin{array}{llll}31.05 & 0.59 & \mathrm{C}_{19} \mathrm{H}_{36} \mathrm{O}_{3} & 312\end{array}$

$\begin{array}{llll}31.86 & 0.63 & \mathrm{C}_{27} \mathrm{H}_{44} \mathrm{O}_{3} & 416\end{array}$ 


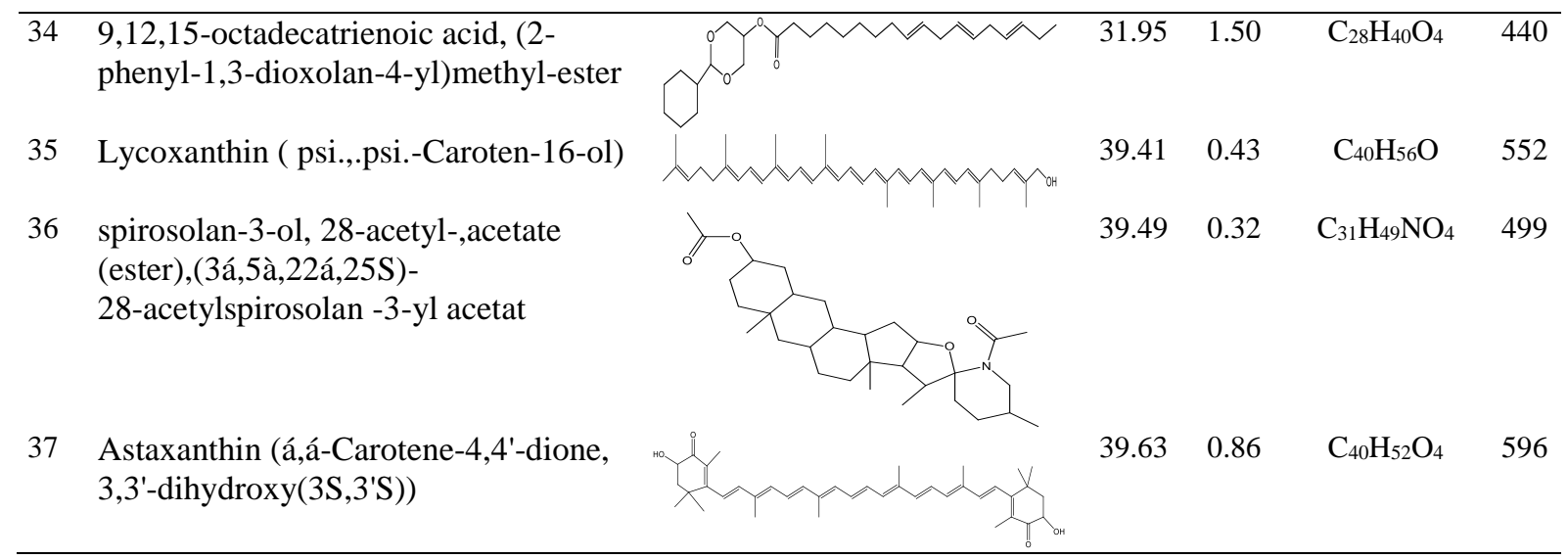

Table 3: Methanolic extract components of Spirulina platensis

\begin{tabular}{|c|c|c|c|c|c|c|}
\hline $\mathbf{N}$ & Compound Name & CSBC & RT & Area \% & M F & MW \\
\hline 1 & Docasane & & 16.63 & 0.66 & $\mathrm{C} 22 \mathrm{H} 46$ & 310 \\
\hline 2 & $\begin{array}{l}\text { Hexadecanoic acid, } \\
\text { methyl ester } \\
\text { (Palmitic acid, methyl } \\
\text { ester) }\end{array}$ & & 21.49 & 29.26 & $\mathrm{C} 17 \mathrm{H} 34 \mathrm{O} 2$ & 270 \\
\hline 3 & $\begin{array}{l}\text { 7,10-Hexadecadienoic } \\
\text { acid, methyl ester }\end{array}$ & & 21.68 & 8.29 & $\mathrm{C} 17 \mathrm{H} 30 \mathrm{O} 2$ & 266 \\
\hline 4 & Phytol & & 23.94 & 4.28 & $\mathrm{C} 20 \mathrm{H} 40 \mathrm{O}$ & 296 \\
\hline 5 & $\begin{array}{l}\text { 9-Octadecenoic acid, } \\
\text { methyl ester,(E)- } \\
\text { Oleic acid, methyl } \\
\text { ester }\end{array}$ & & 24.14 & 20.22 & C19H36O2 & 296 \\
\hline 6 & $\begin{array}{l}\text { 9,12-Octadecadienoic } \\
\text { acid (Z,Z)-, methyl } \\
\text { ester } \\
\text { ( Linoleic acid, } \\
\text { methyl ester) }\end{array}$ & & 24.36 & 34.40 & $\mathrm{C} 19 \mathrm{H} 34 \mathrm{O} 2$ & 294 \\
\hline 7 & $\begin{array}{l}9,12,15- \\
\text { Octadecatrienoic acid, } \\
\text { methyl ester, (Z,Z,Z)- } \\
\text { (Linolenic acid, } \\
\text { methyl ester) }\end{array}$ & & 24.75 & 2.89 & $\mathrm{C} 19 \mathrm{H} 32 \mathrm{O} 2$ & 292 \\
\hline
\end{tabular}

The major constituents of Anabaena variabilis methanolic extract, with its retention time (RT), concentration (area \%), the chemical structure of bioactive components (CSBC), molecular formula (MF), and molecular weight (MW) are presented in Table (2) and Figure 4. Thirty seven bioactive compounds were identified. The most dominant compound was Pentadecanoic acid, 14-methyl-,methyl ester which represents $(13.97 \%)$, followed by 12,15 Octadecadienoic acid, methyl ester which represent $10.23 \%$, followed by Hexadecanoic acid, methyl ester (Palmitic acid, methyl ester) acts $8.26 \%$, also Nonadecane (AI3-36122) represent as a percentage $5.69 \%$, thieno [3,4c]pyridine, 1,3,4,7-tetraphenyl represents as $5.20 \%$, and Heptadecane(AI3-36898) act as a percentage $4.85 \%$, followed by 9,12 octadecadienoic acid (Z,Z)-, 2,3dihydroxypropyl ester ( Linolein, 2-mono-) represents as $2.04 \%$.

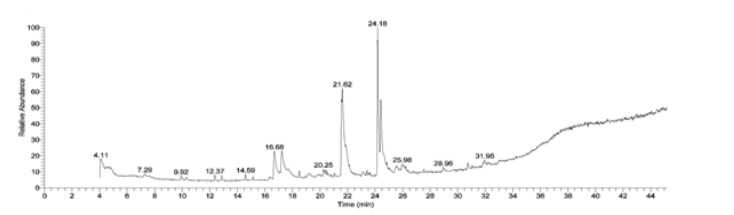

Fig. 4 GC/MS chromatogram of Anabaena variabilis

The major constituents of Spirulina platensis methanolic extract, with its, retention time (RT), concentration (area \%), chemical structure of bioactive components (CSBC), molecular formula (MF), and molecular weight 
(MW) are presented in Table 3 and Figure 5. S. platensis has seven bioactive compounds. The most dominant percentage was ,12Octadecadienoic acid (Z,Z)-, methyl ester (Linoleic acid, methyl ester) with percentage composition of $30.3 \%$ was the major compound followed by Hexadecanoic acid, methyl ester (Palmitic acid, methyl ester) which represents as $29.26 \%$ followed by 9Octadecenoic acid, methyl ester,(E) - Oleic acid, methyl ester as $20.22 \%$. The other compounds are [7, 10-hexadecadienoic acidmethyl ester], [Phytol], [9, 12, 15 Octadecatrienoic acid-methyl ester], and [Docosane] which represent as percentage $8.29 \%, 4.28 \%, 2.89 \%$, and $0.66 \%$, respectively.

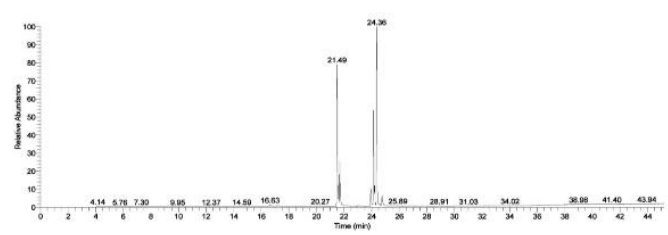

Fig. 5 GC/MS chromatogram of Spirulina platensis

As summarized in Tables 2 and 3, the two analyzed cyanobacterial species have variations in number, type, the arrangement of predominance, and concentration area for each bioactive component. Some strains of cyanobacteria have a high source of bioactive secondary metabolites, which can have therapeutic, industrial, and agricultural importance (Gupta et al. 2013; Rimsha et al. 2014).

Cyanobacteria and algae have various compounds like alkaloids, carbohydrates, flavonoids, pigments, phenols, steroids, and vitamins. (They have applications in many fields as agriculture, pharmaceuticals, biotechnology, and industrial fields. Michalak et al. 2016; Guiheneuf et al. 2016).

In the present study, Octadecane, Heptadecane, 9, 12-Octadecadienoic acid, Linoleic acid methyl ester, Oleic acid methyl ester were among the identified compounds. Octadecanoic acid methyl ester and 9,12,15 Octadecatrienoic acid, 2,3-dihydroxypropyl ester, (Z,Z,Z) (Linolenic acid ester) reported to have activities as antimicrobial, antibacterial, antioxidant, anticancer anti-inflammatory, anti-arthritic, hypocholesterolemic, and cancer preventive (Lee et al. 2007; Mishra and Shree 2007; Wu et al. 2011).

\section{Conclusions}

The present work indicated that, Anabaena variabilis as predominan...........t soil cyanobacterial species, produced 37 bioactive secondary metabolites. While, Spirulina platensis as aquatic cyanobacteria produced only seven phytochemical products. Cyanobacteria extracts have potential bioactive compounds. The total bioactive products, their predominance, and concentration area depend on the specie of Cyanophyta. This work also indicated that the bioactive compounds of $A$. variabilis and $S$. platensis need further research to ascertain their biological properties.

\section{Acknowledgments}

The authors would like Dr. Abdullah Ellattar, Botany Dept., Fac., of Science, Ain Shams University, Cairo, Egypt, for identifying the cyanobacterial strains.

\section{References}

Abed RMM, Dobrestov S, Al-Kharusi S, Schramm A, Jupp B, and Golubic S (2011) Cyanobacterial diversity and bioactivity of inland hypersaline microbial mats from a desert stream in the Sultanate of Oman. Fottea. 11(2011) 215-224.

Agrawal, M., Ghosh, S., and Bagchi, D. (2006) Occurrence of microcystin-containing toxic water blooms in Central India. J Microbiol Biotech. 16(2006): 212-218.

Al-Wathnani H, Ismet A, Tahmaz RR, Al-Dayel $\mathrm{TH}$, and Bakir MA (2012) Bioactivity of natural compounds isolated from cyanobacteria and green algae against human pathogenic bacteria and yeast. J Med Plants Res. 6(18):3425 -3433.

Bethan K, Carole L (2018). Secondary Metabolites in Cyanobacteria, intechopen.75648.

Camila MC, Daniel SM, Jimmy O (2018). The impact of culture conditions on growth and metabolic profiles of freshwater cyanobacteria. $\mathbf{J}$ Appl Phycol. 30(2018):375-384.

Cao, J., Li, X., Kong, X., Zed, R., Dong, L., 2012. Using alfalfa (Medicago sativa) to ameliorate salt-affected soils in Yingda irrigation district in Northwest China. Acta Ecol. Sinica 32 (2), 6873.

Elshouny WA, El-Sheekh MM, Sabae SZ, Khalil MA, Badr HM (2017) Antimicrobial activity of Spirulina platensis against aquatic bacterial isolates. J Microbiol Biotech Food Sci. 6(1) 
1203-1208.

Gheda SF, Ismail GA, (2020) Natural products from some soil cyanobacterial extracts with potent antimicrobial, antioxidant and cytotoxic activities. Annals of the Brazilian Academy of Sciences 29(2):1-18.

Guihéneuf F, Khan A, Tran LSP (2016). Genetic engineering: a promising tool to engender physiological, biochemical, and molecular stress resilience in green microalgae. Front. Plant Sci. 7:400.

Gupta V, Ratha SK, Sood A, Chaudhary V, Prasanna R. (2013). New insights into the biodiversity and applications of cyanobacteria (blue-green algae) Prospects and challenges. Algal Res. 2, 79-97.

Harjinder S, Jasvirinder SK and Amrik SA (2014) Cyanobacteria and agricultural crops. International journal of plant research, 27 (1):3744.

Lau N, Matsui M, Abdullah AA (2015) Cyanobacteria: Photoautotrophic Microbial Factories for the Sustainable Synthesis of Industrial Products. BioMed Research International, 1(2015):1- 9.

Lee, Y.S., and Kang, M.H., (2007) "Effect of constituents of amomun Xanthioides on gastritis in rats and on growth of gastric cancer cell" Arch.Pharm. Res, 30: 436-443.

Lefort-Tran M, Pouphile M, Spathj S, Packer L (1988) Cytoplasmic membrane changes during adaptation of the fresh water Cyanobacterium Synchococcus 6311 to salinity. Plant Physiol.; 87: 767-775.

Michalak I, Chojnacka K, Dmytryk A, Wilk R, Gramza M, Rój E. (2016) Evaluation of supercritical fluid extract of algae as biostimulants of plant growth in field trials. Front. Plant Sci. 2016, 7, 1591.

Mishra PM, Shree A (2007) Antibacterial activity and GC MS analysis of the extract of leaves of Finlaysonia obovata (A Mnagrove Plant) Asi.J.Pl.Sci. 6: 168-172.

Patra JK, Rath SK, Jena K, Rathod VK, Thatoi HN (2008) Evaluating of antioxidant and antimicrobial activity of sea weed (Sargassum sp.) extract: A study on inhibition of Glutathione$S$ transferase activity. Turkish Journal of Biology; 32(1) 119-125.

Prakash JW, Marimuthu JA, Jeeva S(2011) Antimicrobial activity of certain fresh-water microalgae from Thambirabrani River, Tamil Nadu, South India. Asian Pac J Trop Bi-omed. 1(2011) 170-173

Radhika D, Veerabahu C, Priya R (2012)
Antibacterial activity of some selected sea-weeds from Gulf of Mannar Coast, South India. Asian J Pharmaceut Clin Res, 5(2012):89-90.

Ramamurthy V, Raveendran S, Thirumeni S, Krishnaveni S (2012) Antimicrobial activity of heterocytic cyanobacteria. Int. J. Adv.Lif.Sci, 1(2012) 32-39.

Richards, L. A., Ed. (1954). "Diagnosis and Improvement of Saline and Sodic Soils," USDA Agric. Handb. 60. USDA, Washington, DC

Rimsha R, Richa J, Sheela K, Shrivastava PN, Manju J. (2014) Bio-active substances of cyanobacteria (Nostoc muscorum), a review. Int J Pharma Sci Res. 5, 320-322.

Rohloff, J., (2015).Analysis of Phenolic and Cyclic Compounds in Plants Using Derivatization Techniques in Combination with GC-MS-Based Metabolite Profiling. Molecules 20, 3431-3462.

Seddek NH, Fawzy MA, El-Said WA, Ahmed MMR (2019) Evaluation of antimicrobial, antioxidant and cytotoxic activities and characterization of bioactive substances from freshwater blue-green algae, 21(3):328-336.

Skulberg, O. M; (2000) Microalgae as a source of bioactive molecules- experience from cyanophyte research. Journal of Applied Phycology. 12(3):341-348.

Starr TJ, Dieg EF, Church KK, Allen MB, (1962) Antibacterial and antiviral activities of algal extracts studies by acridine orange staining. Texas Report on Biology and Medicine 20: 271278.

Swain DL, Singh DE, Horton DE, Mankin JS, Ballard, TC and Diffenbaugh NS (2017) Remote linkages to anomalous winter atmospheric ridging over the northeastern Pacific. J. Geophys. Res. Atmos, 122(22):12194-12209.

Varsha S and Meeta B (2019) GC/MS Analysis of Bio-active Compounds in Ethanolic Extract of Leaf and Stem of Asclepias curassavica L, Int. J. Pharm. Investigation, 9(2):67-70.

Weggler-Beaton, K., McLaughlin, M.J., Graham, R.D., 2000. Salinity increases cadmium uptake by wheat and Swiss chard from soil amended with biosolids. Aust. J. Soil Res. 38 (1), 37-46.

Wu Y, Liu J, Yang L, Chen H, Zhang S, Zhao H, Zhang $N$ (2011). Allelopathic control of cyanobacterial blooms by periphyton biofilms. Environmental Microbiology, 13: 604-615.

Yadav S, Agrawal M, Raipuria N, Agrawa MK (2016) Antimicrobial activity of Nostoc calcicola (cyanobacteria) iso//lated from Central India against human pathogens. Asian J Pharm. 10(2016):554-559. 


\section{عنوان البحث: المركبات الكيميائية لسلالتين من السيانوبكتريا المحلية أحمد' على دياب'، مصطفى محمد الثيخّ، ريهام صلاح حسن"، عبد الجواد يوسف السعدنى"، سهام السيد أبو

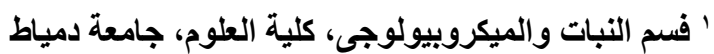

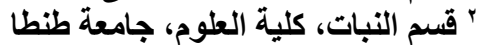

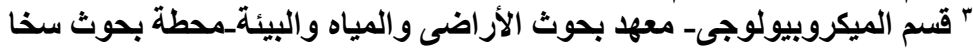

الملخص العربي

تم تجميع طحلب ال Anabaena variabilis من التربة الزر اعية بكفر الثيخ حيث تمت دراسة الظروف البيئية

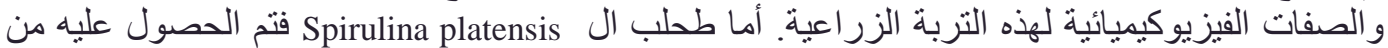

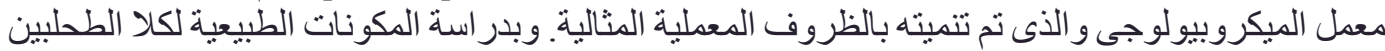

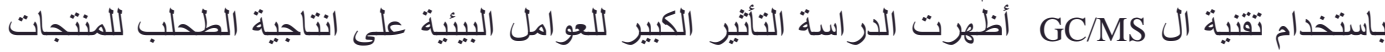

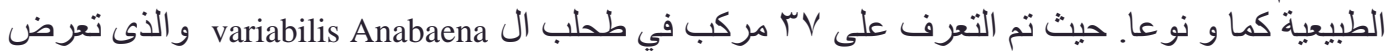

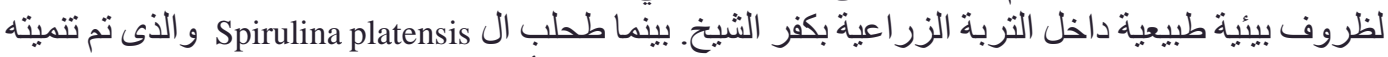

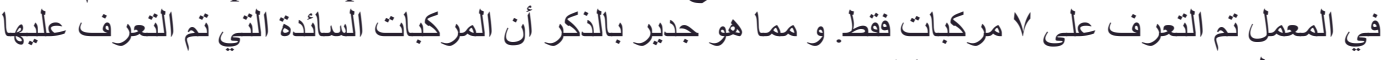

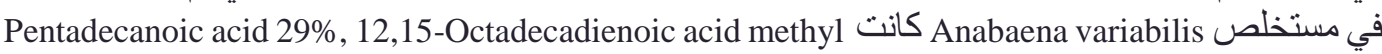
ester 10.26\%, Hexadecenoic acid methyl ester (Z) 8.3\%, Thieno > [3,4-C] pyridine,1,3,4,7tetraphenyl 5.86\% Hexadecanoic acid 29\%, 9,12,15-Octadecadienoic acid methyl ester 24\% and 9,12 Octadecatrienoic acid zz methyl ester, 24.36\% التربة يختلف كما و نو عاو سيادة عن تللك المنتجات لطحالب السيانوبكتريا المعملية. 